\title{
Detection of hepatitis B virus isolates with mutations associated with immune escape mutants among pregnant women in Ibadan, southwestern Nigeria
}

Temitope Oluwasegun Cephas Faleye ${ }^{1,7}$, Moses Olubusuyi Adewumi ${ }^{{ }^{*}}$, ljeoma Maryjoy Ifeorah², Ewean Chukwuma Omoruyi ${ }^{4}$, Solomon Adeleye Bakarey ${ }^{5}$, Adegboyega Akere ${ }^{3}$, Funmilola Awokunle ${ }^{8}$, Hannah Opeyemi Ajibola ${ }^{7}$, Deborah Oluwaseyi Makanjuola ${ }^{8}$ and Johnson Adekunle Adeniji 1,6

\begin{abstract}
Perinatal transmission of hepatitis B virus (HBV) and its associated immune escape mutants (IEMs), is the major vehicle through which a population of chronically infected people who serve as infectious HBV reservoirs is maintained in communities. Therefore, to assess the risk of perinatal transmission, 272 pregnant women attending ante-natal clinics in Ibadan metropolis, southwestern, Nigeria, were screened for HBsAg using ELISA technique. Samples positive for HBsAg were subjected to HBV DNA detection by PCR amplification of the S-gene and amplicon sequencing. Isolates were genotyped and subtyped using a combination of molecular techniques.

Fifteen (5.5\%) of the pregnant women were positive for HBsAg of which HBV DNA was detected in seven. Five of the isolates were typed as genotype E subtype ayw4 using amino acid residues at positions 122, 127, 134 and 160. Another could only be typed as genotype E subtype ayw4 by further phylogenetic analysis. The remaining one isolate did not belong to any of genotypes $\mathrm{A}-\mathrm{H}$. Three of the HBV isolates including the untypable, had mutations in the 'a' determinant associated with IEMs.

This study confirms the endemicity of HBV, the risk of perinatal transmission and the circulation of genotype E subtype ayw4 in Nigeria. It further demonstrates the presence of IEMs in Nigeria.
\end{abstract}

Keywords: HBV; Immune escape mutant; Nigeria; Pregnant women; Vaccine escape mutant

\section{Introduction}

Hepatitis B virus (HBV) belongs to the genus orthohepadnavirus in the family Hepadnaviridae. HBV is an enveloped virus with a diameter of $\sim 42 \mathrm{~nm}$. Within the core of the virus is a protein-linked, $\sim 3.2 \mathrm{~kb}$ DNA genome that is partly double stranded. The HBV genome has four open reading frames (ORFs) (X, S, P and $\mathrm{C}$ ) with the $\mathrm{X}$ and $\mathrm{C}$ ORFs partially overlapping the $\mathrm{P}$ ORF which also has the $\mathrm{S}$ ORF within it but in a different reading frame. Genotypes A - H of HBV have been described (Schaefer 2007) with members of a genotype not differing by more than $8 \%$ of their genome (Okamoto et al. 1988). Sub-genotypes have

\footnotetext{
* Correspondence: adewumi1@hotmail.com

${ }^{1}$ Department of Virology, College of Medicine, University of Ibadan, Ibadan,

Oyo State, Nigeria

Full list of author information is available at the end of the article
}

also been described with members not differing by more than $4 \%$ of their genome (Norder et al. 2004).

Hepatitis B virus (HBV) infection is one of the top 10 viral infections globally (Perz et al. 2006). Serologic evidence of past or present HBV infection has been shown in approximately one-third of the world's population (i.e., over 2 billion people) (World Health Organization 2009). Infection with HBV could result in outcomes ranging from an acute, self-limiting disease through chronic hepatitis $\mathrm{B}(\mathrm{CHB})$ to cirrhosis and hepatocellular carcinoma (HCC) (Gerlich 2013). Furthermore, about 360 million people globally are chronically infected with HBV (World Health Organization 2009).

About $90 \%$ of children who get infected perinatally become chronic HBV carriers (Beasley et al. 1981; Chang 2007). Hence, vertical transmission of HBV is a major 
vehicle through which a reservoir of infectious HBV is maintained in populations (Beasley et al. 1981; Chang 2007). This can and is being addressed by identifying and treating HBV infected pregnant women and administering both passive (Beasley et al. 1981) and active (Poovorawan et al. 1989; Lavanchy 2012) immunization to their offspring at birth. However, there have been cases in which children or adults who have been vaccinated against $\mathrm{HBV}$ and have serologic correlates of HBV immunity get re-infected with HBV (Carman et al. 1990; Harrison et al. 1991; Fujii et al. 1992; Theamboonlers et al. 2001). The HBV isolates recovered from such cases are usually referred to as Immune Escape Mutants (IEMs) (Carman et al. 1990; Lada et al. 2006; Ramezani et al. 2013).

Immune escape mutants (IEMs) were first described in Italy in 1990 (Carman et al. 1990). The development of IEMs has been ascribed to the fact that HBV has both an RNA phase in its replication cycle and a polymerase without 'proof-reading' ability, and as such has an error rate that is close to that of RNA viruses (Orito et al. 1989; Mizokami and Orito 1999). Consequently, mutants develop spontaneously and can be selected for by antiHBs and the use of nucleoside/nucleotide analogue classes of antiviral drugs (Cento et al. 2013).

Several studies have reported the presence and circulation of IEMs (Carman et al. 1990; Harrison et al. 1991; Fujii et al. 1992; Theamboonlers et al. 2001; Forbi et al. 2013) but not in Nigeria. Since HBV vaccination was added to the National Immunization programme over ten years ago (WHO 2005a; Sadoh and Eregie 2008) and nucleoside/nucleotide analogue classes of antiviral drugs are being used by the HIV and/or HBV infected population in the country (WHO 2005b), this study was designed to investigate the possible emergence and circulation of IEMs in pregnant women in Nigeria. Here we report, for the first time, the presence of HBV IEMs in Nigeria.

\section{Material and methods Study location}

This study was carried out among pregnant women attending two different ante-natal clinics in Ibadan, southwestern Nigeria. The two hospitals were selected to facilitate true representation of the population in the study. The first being the University College Hospital (UCH); a tertiary health care facility of the University of Ibadan. The second hospital is Ade-Oyo State Hospital (ASH); a secondary health care facility located in a central, densely populated part of the city. Attendees in both ante-natal clinics are majorly residents in the city.

\section{Enrolment of patients}

Consenting ante-natal clinic attendees were enrolled from the two selected hospitals described above. Enrolment took place between September 2012 and June, 2013.
During the period, a total of 813 and 1686 ante-natal clinic attendees were enrolled at the ASH and UCH respectively. Short presentation on HBV prevalence and prevention was given during each visit to the antenatal clinics. Thereafter, consenting clinic attendees were enrolled for the study. Demographic and other relevant information were retrieved from the study participants using a structured questionnaire. Afterwards, blood sample was collected from each of the 272 \{median age $=$ 31.07 years, age range $=17-43$ years (NGRAD: $n=90$; age range $=19-42$ years; NGRUC: $n=182$; age range $=17-43$ years)\} consenting subjects enrolled at the point of registration and routine examination for ante-natal clinic. Ethical approvals for the study were granted by the UI/UCH Ethics Committee (UI/EC/11/0058) and Ministry of Health (AD3/479/349).

\section{Sample collection}

Five milliliters of blood was collected from each pregnant woman by venepuncture. The blood sample was then dispensed into an appropriately labeled sterile container without any preservative or anticoagulant. Subsequently, the samples were transported to the laboratory at about $4-8^{\circ} \mathrm{C}$ in a cooler with frozen ice packs. Serum was separated from other blood components by low-speed centrifugation at $500 \mathrm{~g}$ for 5 minutes and subsequently removed using a sterile disposable pipette. Two aliquots of serum were made per sample in labeled sterile cryovials which were stored at $-20^{\circ} \mathrm{C}$ until ready for analysis. Laboratory analysis was carried out in the Department of Virology, and the Institute for Advanced Medical Research and Training, College of Medicine, University of Ibadan, Ibadan, Nigeria.

\section{HBsAg ELISA screen}

All the 272 samples were subjected to HBsAg specific Enzyme Linked Immunosorbent Assay (ELISA) using the HBsAg detection ELISA kit (Diagnostic Automation/ Cortez Diagnostic, California, USA). The assay was performed according to manufacturer's instructions. The optical density was read using the Emax endpoint ELISA microplate reader (Molecular Devices, California, USA) and the result was interpreted according to the manufacturer's instructions.

\section{DNA extraction and HBsAg specific Polymerase Chain Reaction (PCR)}

DNA was extracted using the QIAGEN DNA extraction kit (Qiagen, Hilden, Germany) according to the manufacturer's instructions. Subsequently, a nested PCR assay targeting a $\sim 408$ bp stretch within the S ORF was used to detect HBV DNA. First round primers were HBV_S1F 5-CTAGGACCCCTGCTCGTGTT-3, and HBV_S1R 5CGAACCACTGAACAAATGGCACT-3, while second round primers were HBV_SNF 5-GTTGACAAGAATCC 
TCACAATACC-3 and HBV_SNR 5-GAGGCCCACTC CCATA-3 (Forbi et al. 2013). Primers were made in $25 \mu \mathrm{M}$ concentrations and two microliter of each of the primers was added to a $50 \mu \mathrm{L}$ reaction containing $10 \mu \mathrm{L}$ of Red load Taq (Jena Bioscience, Jena, Germany), $4 \mu \mathrm{L}$ of DNA and $32 \mu \mathrm{L}$ of RNase free water. Thermal cycling was done in a Veriti Thermalcycler (Applied Biosystems, California, USA.) as follows; $94^{\circ} \mathrm{C}$ for 3 minutes followed by 45 cycles of $94^{\circ} \mathrm{C}$ for 30 seconds, $55^{\circ} \mathrm{C}$ for 60 seconds and $70^{\circ} \mathrm{C}$ for 40 seconds with ramp of $40 \%$ from $55^{\circ} \mathrm{C}$ to $70^{\circ} \mathrm{C}$. This was then followed by $72^{\circ} \mathrm{C}$ for 7 minutes and held at $4^{\circ} \mathrm{C}$ till terminated. Reaction conditions were the same for both first and second round PCR except that DNA extract from the sample was used as template for first round PCR while first round PCR product was used as template for second round PCR. Finally, PCR products were resolved on $2 \%$ agarose gels stained with ethidium bromide and viewed using a UV transilluminator.

\section{Amplicon sequencing}

The PCR reactions that were positive by having the required amplicon size were shipped to Macrogen Inc, Seoul, South Korea, for amplicon purification and BigDye chemistry sequencing. Sequencing was done using primers HBV_SNF and HBV_SNR.

\section{Phylogenetic analysis}

Amino acid residues at positions 122, 127, 134 and 160 were initially used to determine HBV genotypes and serotypes (Forbi et al. 2013). Subsequently, HBsAg reference sequences were retrieved from the HBV database (http://hbvdb.ibcp.fr/HBVdb/) and aligned using the CLUSTAL W program in MEGA 5 software with default settings (Tamura et al. 2011). Afterwards, a neighborjoining tree was constructed using MEGA 5 software with the Kimura-2 parameter model (Kimura 1980) and 1,000 bootstrap replicates. Furthermore, pairwise distance of the $\mathrm{HBsAg}$ sequences were estimated using MEGA 5 software with Kimura-2 parameter model (Kimura 1980). The accession numbers of sequences retrieved from $\mathrm{HBV}$ database for phylogenetic analysis are indicated in the sequence names on the phylograms.

\section{Nucleotide sequence accession numbers}

The sequences reported in this study have been submitted to GenBank Nucleotide Sequence Database under the accession numbers KM225621 - KM225627.

\section{Results}

HBsAg ELISA screen, DNA extraction and Surface/Pol gene specific Polymerase Chain Reaction

A total of 15 (5.5\%) of the 272 samples were positive for HBsAg. DNA was extracted from all the 15 samples that were positive for the HBsAg ELISA screen, and subjected to HBsAg specific PCR. Despite repeated attempts the $\sim 408$ bp amplicon within the S ORF was successfully amplified in only 7 (46.7\%) of the $15 \mathrm{HBsAg}$ positive samples.

\section{Phylogenetic analysis}

Based on the amino acid residues at positions s122, s127, s134 and s160 (Forbi et al. 2013), five of the seven sequenced isolates belonged to genotype $\mathrm{E}$ and serotype ayw4 (Table 1). The remaining two HBV isolates could not be typed using this classification scheme because isolate NGRUC-12-054-A8-HBsAg had sR122Q substitution and isolate NGRUC-13-100-C1-HBsAg had sF134V substitution (Figure 1). To classify the two untypable HBV isolates, a phylogram was constructed using the sequences isolated in this study alongside reference HBV sequences of genotypes $\mathrm{A}-\mathrm{H}$ from the HBV database (http://hbvdb. ibcp.fr/HBVdb/). The phylogram (Figure 2) showed that, just like the previously classified five isolates, isolate NGRUC-13-100-C1-HBsAg belonged to genotype E, ayw4. Furthermore, isolate NGRUC-13-100-C1-HBsAg is only $1.1 \%$ divergent (Table 2 ) from a reference genotype $\mathrm{E}$, ayw4 strain (AB091255). Hence, confirming its membership in this group.

The phylogram (Figure 2) also showed that isolate NGRUC-12-054-A8-HBsAg, did not belong to any of

Table 1 Serotype and genotype of sequenced HBV isolates

\begin{tabular}{|c|c|c|c|c|c|c|c|}
\hline \multirow[t]{2}{*}{$\mathrm{S} / \mathrm{N}$} & \multirow[t]{2}{*}{ Sample ID } & \multicolumn{4}{|c|}{$\begin{array}{l}\text { Amino acid residues at positions within the } \\
\text { surface antigen for serotype determination }\end{array}$} & \multirow[t]{2}{*}{ Serotype } & \multirow[t]{2}{*}{ Genotype } \\
\hline & & $s 122$ & s127 & s134 & $s 160$ & & \\
\hline 1 & NGRAD-13-065-A4-HBsAg & $\mathrm{R}$ & L & $\mathrm{F}$ & K & ayw4 & E \\
\hline 2 & NGRUC-12-054-A8-HBsAg & Q & L & $\mathrm{F}$ & K & Untypable & Untypable \\
\hline 3 & NGRUC-13-008-B2-HBsAg & $\mathrm{R}$ & L & F & K & ayw4 & E \\
\hline 4 & NGRUC-13-045-M8-HBsAg & $\mathrm{R}$ & L & $\mathrm{F}$ & K & ayw4 & $E$ \\
\hline 5 & NGRUC-13-078-B6-HBsAg & $\mathrm{R}$ & $\mathrm{L}$ & F & K & ayw4 & $E$ \\
\hline 6 & NGRUC-13-084-B7-HBsAg & $\mathrm{R}$ & L & $\mathrm{F}$ & K & ayw4 & E \\
\hline 7 & NGRUC-13-100-C1-HBsAg & $\mathrm{R}$ & L & V & K & Untypable & Untypable \\
\hline
\end{tabular}




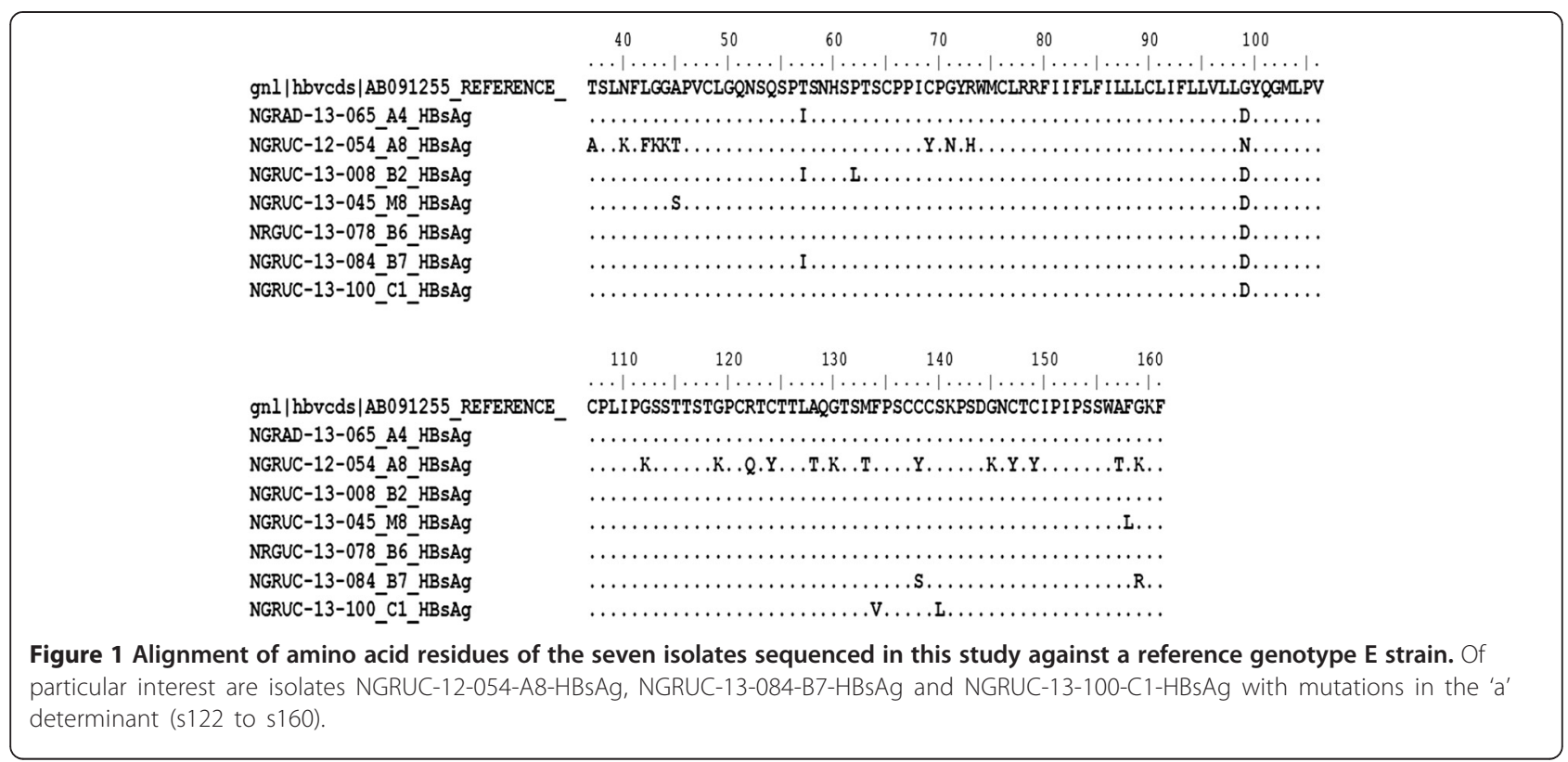

genotypes A - H. Rather, it stood apart as an outgroup. Similarity matrix (Table 3 ) also showed that it is between 12.0 and $18.9 \%$ divergent from genotypes $\mathrm{A}-\mathrm{H}$ and as such does not belong to any of the genotypes. It is however most closely related to genotype $\mathrm{E}$ at $12.0 \%$. The Major Hydrophilic Region (MHR; s122 - s160) of isolate NGRUC-12-054-A8-HBsAg showed an abundance of substitutions which included; sG112K, sG119K, sR122Q, sC124Y, sA128T, sG130K, sM133T, sC138Y, sG145K, sC147Y, sC149Y, sA157T and sG159K (Figure 1).

Other isolates asides NGRUC-12-054-A8-HBsAg also have significant substitutions within the MHR. These included, isolates NGRUC-13-084-B7-HBsAg (sC138S and sG159R) and NGRUC-13-100-C1-HBsAg (sF134V and sS140L) (Figure 1).

\section{Discussion}

\section{Mutations in the $\mathrm{S}$ gene and vaccine escape mutants}

The results of this study showed the presence of G145K in one of the HBV isolates (NGRUC-12-054-A8-HBsAg) detected. This substitution alongside G145R have been associated with immune escape mutants (IEMs) (Carman et al. 1990, 1995; Karthigesu et al. 1994; Grethe et al. 1998; Lada et al. 2006). This study therefore reports for the first time the presence of IEMs in Nigeria and particularly in a pregnant woman.

Asides the G145K substitution, NGRUC-12-054-A8HBsAg had other substitutions that might work synergistically to enhance the immune escape phenotype predicted. For example, the substitution of cysteine residues at positions s124, s138, s147 and s149 with tyrosine (Figure 1) will abolish the formation of the disulphide bonds predicted to stabilise the double-loop structure of the HBsAg extravirion region (Prange and Streeck 1995). Insilico structure prediction studies (unpublished data) suggested that the three dimensional conformation of the extravirion loop of this isolate is different from that of the wild-type genotype E, ayw4 isolate. In addition, instead of being extravirion, the loop is embedded in the polar head-groups of the extravirion leaflet of the virion membrane. This buttresses the fact that this isolate might indeed be an IEM. Similar structural prediction results were obtained for isolates NGRUC-13-084-B7-HBsAg and NGRUC-13-100-C1-HBsAg which also have substitutions between residues $\mathrm{s} 133$ and $\mathrm{s} 144$ (unpublished data).

The results of this study showed that 3 out of the 7 isolates (NGRUC-12-054-A8-HBsAg, NGRUC-13-084B7-HBsAg and NGRUC-13-100-C1-HBsAg) sequenced in this study had mutations that have been associated with immune escape mutants (IEMs) (Carman et al. 1990, 1995; Karthigesu et al. 1994; Grethe et al. 1998; Lada et al. 2006; Forbi et al. 2013). This may imply considerable rate of IEM emergence. However, the ecological system necessary to create the adequate amount of evolutionary pressure necessary to select for HBV IEMs at such high rate is present in Nigeria. Factors necessary to provide such selective pressure include spontaneous errors by HBV polymerase, the host immune response to natural HBV infection, active and passive immunization, and use of nucleoside/nucleotide analogue antiviral drugs by HIV and/or HBV infected individuals (Cento et al. 2013). The first two factors are intrinsic to HBV and humans, and are consequently always present while, the last two have been in place in Nigeria for over ten years 


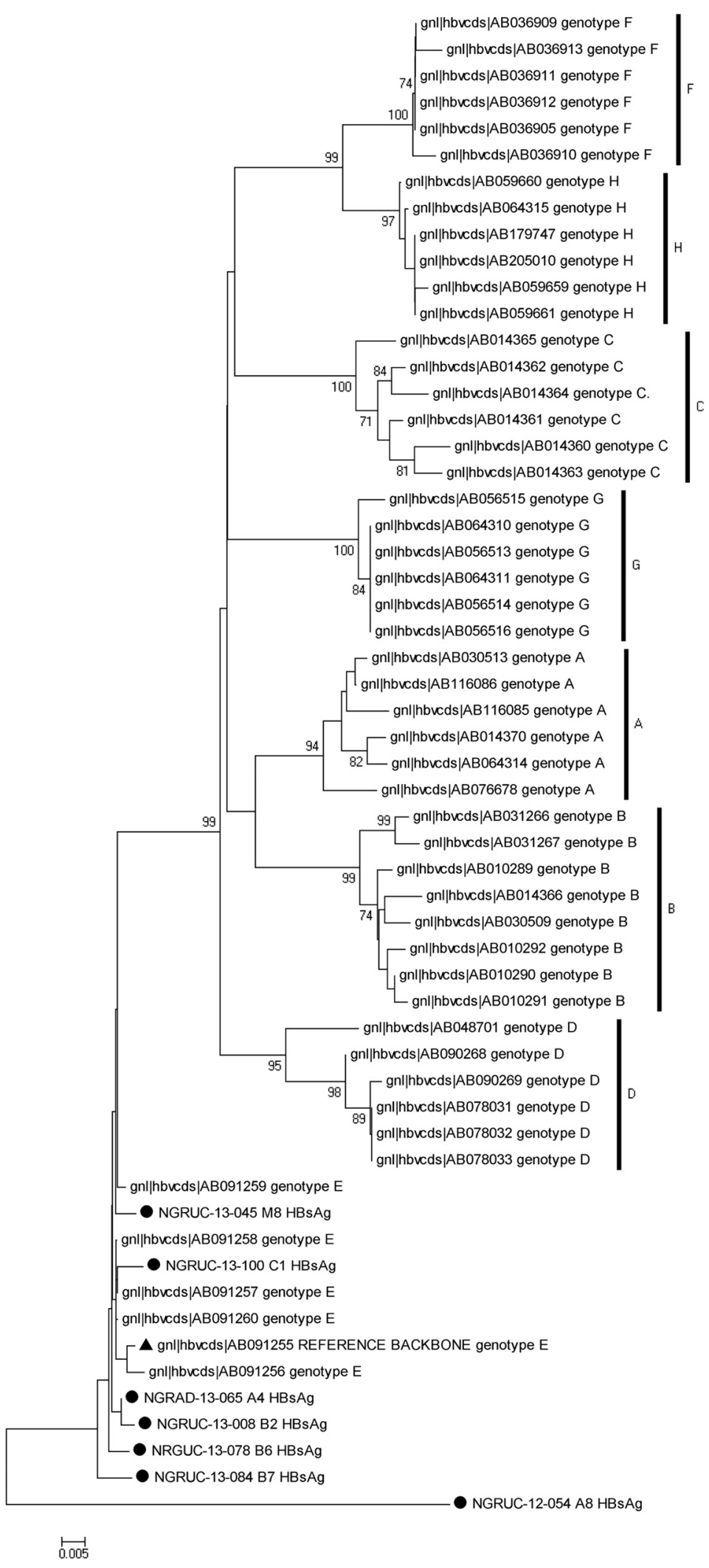

Figure 2 Phylogenetic relationship of recovered HBV isolates. The phylogram is based on an alignment of the partial HBsAg sequences. The newly sequenced strains are highlighted with black circle, while a genotype E reference strain is highlighted with a black triangle. The GenBank accession number of the strains are indicated in the tree. Bootstrap values are indicated if $\geq 70 \%$. 
Table 2 Comparison of reference sequences gnl|hbvcds|AB091255 genotype $E$ to all isolates recovered in this study

\begin{tabular}{|c|c|c|c|}
\hline Species 1 & Species 2 & Dist \% & Siml \% \\
\hline gnl|hbvcds|AB091255 REFERENCE BACKBONE genotype E & NGRAD-13-065 A4 HBsAg & 0.8 & 99.2 \\
\hline gnl|hbvcds|AB091255 REFERENCE BACKBONE genotype E & NGRUC-12-054 A8 HBsAg & 12.5 & 87.5 \\
\hline gnl|hbvcds|AB091255 REFERENCE BACKBONE genotype E & NGRUC-13-008 B2 HBsAg & 1.1 & 98.9 \\
\hline gnl|hbvcds|AB091255 REFERENCE BACKBONE genotype E & NGRUC-13-045 M8 HBsAg & 1.1 & 98.9 \\
\hline gnl|hbvcds|AB091255 REFERENCE BACKBONE genotype E & NRGUC-13-078 B6 HBsAg & 1.1 & 98.9 \\
\hline gnl|hbvcds|AB091255 REFERENCE BACKBONE genotype E & NGRUC-13-084 B7 HBsAg & 1.6 & 98.4 \\
\hline gnl|hbvcds|AB091255 REFERENCE BACKBONE genotype E & NGRUC-13-100 C1 HBsAg & 1.1 & 98.9 \\
\hline gnl|hbvcds|AB091255 REFERENCE BACKBONE genotype E & gnl|hbvcds|AB091260 genotype $E$ & 0.5 & 99.5 \\
\hline
\end{tabular}

(WHO 2005a, b; Sadoh and Eregie 2008;). This therefore shows that the conditions had always been present for HBV IEMs to emerge.

The HBV IEMs characterized in this study did not share mutations in the 'a' determinant (Figure 1). This shows that the IEMs must have emerged as a result of independent events. This finding has two implications. Firstly, it corroborates the fact that the conditions were perfect for HBV IEM emergence in Nigeria, and secondly, it shows that HBV immune escape mutants can evolve over a sequence and conformational space.
However, as a result of the small number of isolates recovered in this study, it is difficult to extrapolate as regards the circulation of these mutants. What is obvious is that the results of this study do not provide evidence to support circulation of these mutants in the region and this is corroborated by the fact that none of the previous studies in the region ever reported detection of HBV IEMs (Odemuyiwa et al. 2001; Forbi et al. 2010).

It is pertinent to note that, despite repeated attempts the 408 bp amplicon within the S ORF could not be

Table 3 Comparison of isolate NGRUC-12-054 A8 HBsAg to all isolates recovered in this study and reference sequences from genotypes $\mathrm{A}$ to $\mathrm{H}$

\begin{tabular}{|c|c|c|c|}
\hline Species 1 & Species 2 & Dist \% & Siml \% \\
\hline NGRUC-12-054 A8 HbsAg & NGRUC-13-008 B2 HBsAg & 12.6 & 87.4 \\
\hline NGRUC-12-054 A8 HbsAg & NGRUC-13-045 M8 HBsAg & 12.2 & 87.8 \\
\hline NGRUC-12-054 A8 HbsAg & NRGUC-13-078 B6 HBsAg & 12.0 & 88.0 \\
\hline NGRUC-12-054 A8 HbsAg & NGRUC-13-084 B7 HBsAg & 12.2 & 87.8 \\
\hline NGRUC-12-054 A8 HbsAg & NGRUC-13-100 C1 HBsAg & 12.6 & 87.4 \\
\hline NGRUC-12-054 A8 HbsAg & gnl|hbvcds|AB116085 genotype A & 18.2 & 81.8 \\
\hline NGRUC-12-054 A8 HbsAg & gnl|hbvcds|AB116086 genotype A & 16.8 & 83.2 \\
\hline NGRUC-12-054 A8 HbsAg & gnl|hbvcds|AB031266 genotype B & 18.2 & 81.8 \\
\hline NGRUC-12-054 A8 HbsAg & gnl|hbvcds|AB031267 genotype B & 17.8 & 82.2 \\
\hline NGRUC-12-054 A8 HbsAg & gnl|hbvcds|AB014364 genotype C. & 18.9 & 81.1 \\
\hline NGRUC-12-054 A8 HbsAg & gnl|hbvcds|AB014365 genotype C & 17.2 & 82.8 \\
\hline NGRUC-12-054 A8 HbsAg & gnl|hbvcds|AB090268 genotype D & 17.3 & 82.7 \\
\hline NGRUC-12-054 A8 HbsAg & gnl|hbvcds|AB090269 genotype D & 18.4 & 81.6 \\
\hline NGRUC-12-054 A8 HbsAg & gnl|hbvcds|AB091260 genotype E & 12.0 & 88.0 \\
\hline NGRUC-12-054 A8 HbsAg & gnl|hbvcds|AB036912 genotype $F$ & 18.2 & 81.8 \\
\hline NGRUC-12-054 A8 HbsAg & gnl|hbvcds|AB036913 genotype $F$ & 18.9 & 81.1 \\
\hline NGRUC-12-054 A8 HbsAg & gnl|hbvcds|AB064310 genotype G & 17.6 & 82.4 \\
\hline NGRUC-12-054 A8 HbsAg & gnl|hbvcds|AB064311 genotype G & 17.6 & 82.4 \\
\hline NGRUC-12-054 A8 HbsAg & gnl|hbvcds|AB179747 genotype H & 18.2 & 81.8 \\
\hline NGRUC-12-054 A8 HbsAg & gnl|hbvcds|AB205010 genotype H & 18.2 & 81.8 \\
\hline NGRUC-12-054 A8 HbsAg & NGRAD-12-065 A4 HBsAg & 12.2 & 87.8 \\
\hline NGRUC-12-054 A8 HbsAg & Gnl|hbvcds|AB091255 reference backbone genotype E & 12.5 & 87.5 \\
\hline
\end{tabular}


amplified in 8 (53.3\%) of the $15 \mathrm{HBsAg}$ positive samples. This could be due to low viral load, primer mismatch as a result of point mutations or virus strains being of recombinant origin. However, effort is underway to further characterize the isolates using sequence independent strategies.

\section{HBV genotypes circulating in Nigeria and the classification scheme}

The circulation in Nigeria of HBV genotype E, serotype ayw4 was documented in this study. This confirms previous reports (Odemuyiwa et al. 2001; Forbi et al. 2010). Furthermore, the findings of this study confirm the inclusion of Nigeria in the HBV genotype E crescent as previously described (Odemuyiwa et al. 2001; Mulders et al. 2004; Olinger et al. 2006; Andernach et al. 2009; Forbi et al. 2010). However, the use of amino acid residues at positions s122, s127, s134 and s160, though useful for classifying HBV isolates, obviously has its limitations. For example, of the six HBV genotype E, serotype ayw4 isolates sequenced in this study, five could be typed as such using this algorithm. This algorithm failed to identify the last isolate because of an sF134V substitution. Had it not been for phylograms and similarity matrices one of the isolates would have gone untyped. It might therefore be necessary to combine methods when identifying HBV isolates. Also, the inclusion of valine as one of the amino acid residues that could be present at position 134 of $\mathrm{HBV}$ genotype E, ayw4 should be considered.

\section{HBsAg prevalence in pregnant women}

In this study, HBsAg prevalence of 5.5\% was observed among pregnant women in southwestern Nigeria. This is similar to a prevalence of $6 \%$ found in south-south Nigeria (Alegbeleye et al. 2013) but different from higher prevalence $(>8 \%)$ reported in other parts of the country (Ndams et al. 2008; Olokoba et al. 2011). The reasons for this variation is not clear, though several reasons have been suggested (Alegbeleye et al. 2013). Given HBsAg prevalence of $5.5 \%$ (as the lower limit) among pregnant women in Nigeria, an annual birth cohort of 7,310,490 in Nigeria (Gavi Alliance 2014), and a $<5 \%$ risk of developing $\mathrm{CHB}$ in children that acquire HBV perinatally from $\mathrm{HBsAg}$ positive but $\mathrm{HBeAg}$ negative mothers (Hsu et al. 2004; Chang 2007). This implies that about 20,104 children on the average might be developing CHB annually in Nigeria.

As observed in Taiwan (Su et al. 2012) and Thailand (Poovorawan et al. 2011), with the incorporation of HBV vaccine into the National Immunization Programme and significant vaccine coverage, the number of children developing $\mathrm{CHB}$ annually should reduce to about $0.5 \%$ (i.e., 101 $\mathrm{CHB}$ children in Nigeria) of the estimated present value in about two decades. However, in Nigeria, though HBV vaccine is administered at birth in tertiary health care facilities, the same cannot be said of secondary and primary health care facilities. Consequently, reduction in the number of children estimated to develop CHB to about $0.5 \%$ of the exposed may not have been achieved in the country. Though, the possibility of achieving the above stated still exist, since the country still has about a decade (WHO 2005a; Sadoh and Eregie 2008) to attain comparable situation with Taiwan (Su et al. 2012) and Thailand (Poovorawan et al. 2011).

Even if the National Immunization Programme in Nigeria achieves the reduction in estimated number of children developing $\mathrm{CHB}$ to $0.5 \%$ of the exposed, the presence of HBV IEMs and probable perinatal transmission to about $6.7 \%$ (one IEM out of $15 \mathrm{HBsAg}$ positive; a lower limit of this study) of the perinatally exposed might undermine the impact of the vaccination effort by the immunization being ineffective in 1347 children. This might result in the development of a cohort of $\mathrm{CHB}$ children that may serve as reservoirs for the maintenance of HBV, and specifically HBV IEMs in the population.

\section{Conclusion}

The results of this study showed, for the first time in Nigeria, the presence of HBV isolates with mutations that have been associated with immune escape mutants. The results also confirmed circulation of HBV IEMs among pregnant women in Nigeria.

\section{Competing interests}

The authors declare that they have no competing interest.

\section{Authors' contributions}

The study was designed by FTOC, AMO, IIM, OEC and BSA. Sample collection was by AMO, IIM, OEC and AA. Laboratory analysis was by FTOC, AMO, IIM, $\mathrm{OEC}, \mathrm{BSA}, \mathrm{AF}, \mathrm{AHO}$ and $\mathrm{MDO}$. All authors contributed reagents used for the study. FTOC wrote the first draft of the manuscript, and all the authors edited the manuscript. All the authors also read and approved the final draft of the manuscript.

\section{Acknowledgement}

The authors thank the subjects who participated in this study. We also thank the members of staff of the ante-natal clinics of both the University College Hospital and Ade-Oyo Maternity Hospital for their support during sample collection. Finally, we thank Professor O. G. Ademowo of the Institute for Advanced Medical Research and Training, University of Ibadan, for providing Laboratory space and equipment used at some point during the execution of this study.

This study was carried out by the Evolutionary Dynamics of Hepatitis in Nigeria $(E D H I N)$ research group. This study was not funded by any organization.

\section{Author details}

${ }^{1}$ Department of Virology, College of Medicine, University of Ibadan, Ibadan, Oyo State, Nigeria. ${ }^{2}$ Department of Surgery, College of Medicine, University of Ibadan, Ibadan, Oyo State, Nigeria. ${ }^{3}$ Department of Medicine, College of Medicine, University of Ibadan, Ibadan, Oyo State, Nigeria. ${ }^{4}$ Institute of Child Health, College of Medicine, University of Ibadan, Ibadan, Oyo State, Nigeria. ${ }^{5}$ Institute for Advanced Medical Research and Training, College of Medicine, University of Ibadan, Ibadan, Oyo State, Nigeria. ${ }^{6} \mathrm{WHO}$ National Polio Laboratory, University of Ibadan, Ibadan, Oyo State, Nigeria. ${ }^{7}$ Department of 
Microbiology, Faculty of Science, Ekiti State University, Ado Ekiti, Ekiti State, Nigeria. ${ }^{8}$ Department of Science Laboratory Technology, Faculty of Science, Ekiti State University, Ado Ekiti, Ekiti State, Nigeria.

\section{Received: 30 August 2014 Accepted: 12 January 2015} Published online: 01 February 2015

\section{References}

Alegbeleye JO, Nyengidiki TK, Ikimalo JI (2013) Maternal and neonatal seroprevalence of hepatitis B surface antigen in a hospital based population in South-South, Nigeria. Int J Med Med Sci 5(5):241-246

Andernach IE, Hubschen JM, Muller CP (2009) Hepatitis B virus: the genotype E puzzle. Rev Med Virol 19:231-240

Beasley RP, Hwang LY, Lin CC, Stevens CE, Wang KY, Sun TS, Hsieh FJ, Szmuness W (1981) Hepatitis B immune globulin (HBIG) efficacy in the interruption of perinatal transmission of hepatitis B virus carrier state. Initial report of a randomised double-blind placebo-controlled trial. Lancet 2(8243):388-393

Carman WF, Zanetti AR, Karayiannis P, Waters J, Manzillo G, Tanzi E, Zuckerman AJ, Thomas HC (1990) Vaccine-induced escape mutant of hepatitis B virus. Lancet 336:325-329

Carman WF, Korula J, Wallace L, MacPhee R, Mimms L, Decker R (1995) Fulminant reactivation of hepatitis $B$ due to envelope protein mutant that escaped detection by monoclonal HBsAg ELISA. Lancet 345:1406-1407

Cento V, Mirabelli C, Dimonte S, Salpini R, Han Y, Trimoulet P, Bertoli A, Micheli V, Gubertini G, Cappiello G, Spanò A, Longo R, Bernassola M, Mazzotta F, De Sanctis GM, Zhang XX, Verheyen J, D'Arminio Monforte A, CeccheriniSilberstein F, Perno CF, Svicher V (2013) Overlapping structure of hepatitis $B$ virus (HBV) genome and immune selection pressure are critical forces modulating HBV evolution. J Gen Virol 94:143-149

Chang MH (2007) Hepatitis B, virus infection. Semin Fetal Neonatal Med 12:160-167

Forbi JC, Vaughan G, Purdy MA, Campo DS, Xia GL, Ganova-Raeva LM, Ramachandran S, Thai H, Khudyakov YE (2010) Epidemic History and Evolutionary Dynamics of Hepatitis B Virus Infection in Two Remote Communities in Rural Nigeria. PLoS One 5(7):e11615, doi:10.1371/journal.pone.0011615

Forbi JC, Ben-Ayed Y, Xia GL, Vaughan G, Drobeniuc J, Switzer WM, Khudyakov YE (2013) Disparate distribution of hepatitis B virus genotypes in four sub-Saharan African countries. J Clin Virol 58:59-66

Fujii H, Moriyama K, Sakamoto N, Kondo T, Yasuda K, Hiraizumi Y, Yamazaki M, Sakaki Y, Okochi K, Nakajima E (1992) Gly 145 to Arg substitution in HBs antigen of immune escape mutant of hepatitis B virus. Biochem Biophys Res Commun 184:1152-1157

Gavi Alliance. http://www.gavialliance.org/country/nigeria/ visited on the 30th July 2014.

Gerlich WH (2013) Medical Virology of Hepatitis B: how it began and where we are now. Virol J 10:239

Grethe S, Monazahian M, Bohme I, Thomssen R (1998) Characterization of unusual escape variants of hepatitis B virus isolated from a hepatitis B surface antigen negative subject. J Virol 72:7692-7696

Harrison TJ, Hopes EA, Oon CJ, Zanetti AR, Zuckerman AJ (1991) Independent emergence of a vaccine-induced escape mutant of hepatitis $B$ virus. J Hepatol 13:105-107

Hsu HY, Chang MH, Ni YH, Chen HL (2004) Survey of hepatitis B surface variant infection in children 15 years after a nationwide vaccination programme in Taiwan. Gut 53:1499-1503

Karthigesu VD, Allison LM, Fortuin M, Mendy M, Whittle HC, Howard CR (1994) A novel hepatitis B virus variant in the sera of immunized children. J Gen Virol 75(Pt 2):443-448

Kimura M (1980) A simple method for estimating evolutionary rate of base substitutions through comparative studies of nucleotide sequences. J Mol Evol 16(2):111-120

Lada O, Benhamou Y, Poynard T, Thibault V (2006) Coexistence of Hepatitis B Surface Antigen (HBs Ag) and Anti-HBs Antibodies in Chronic Hepatitis B Virus Carriers: Influence of "a" Determinant Variants. J Virol 80(6):2968-2975

Lavanchy D (2012) Viral hepatitis: global goals for vaccination. J Clin Virol 55:296-302

Mizokami M, Orito E (1999) Molecular evolution of hepatitis viruses. Intervirology 42:159-165

Mulders MN, Venard V, Njayou M, Edorh AP, Bola Oyefolu AO, Kehinde MO, Muyembe Tamfum JJ, Nebie YK, Maiga I, Ammerlaan W, Fack F, Omilabu SA, Le Faou A, Muller CP (2004) Low genetic diversity despite hyperendemicity of hepatitis B virus genotype E throughout West Africa. J Infect Dis 190:400-408

Ndams IS, Joshua IA, Luka SA, Sadiq HO (2008) Epidemiology of hepatitis B infection among pregnant women in Minna, Nigeria. Sci World J 3(3):5-8

Norder H, Couroucé AM, Coursaget P, Echevarria JM, Lee SD, Mushahwar IK, Robertson BH, Locarnini S, Magnius LO (2004) Genetic diversity of hepatitis $B$ virus strains derived worldwide: genotypes, subgenotypes, and HBsAg subtypes. Intervirology 47:289-309

Odemuyiwa SO, Mulders MN, Oyedele OI, Ola SO, Odaibo GN, Olaleye DO, Muller CP (2001) Phylogenetic analysis of new hepatitis B virus isolates from Nigeria supports endemicity of genotype $E$ in West Africa. J Med Virol 65:463-469

Okamoto H, Tsuda F, Sakugawa H, Sastrosoewignjo RI, Imai M, Miyakawa Y, Mayumi M (1988) Typing hepatitis B virus by homology in nucleotide sequence: comparison of surface antigen subtypes. J Gen Virol 69:2575-2583

Olinger CM, Venard V, Njayou M, Oyefolu AO, Maïga I, Kemp AJ, Omilabu SA, le Faou A, Muller CP (2006) Phylogenetic analysis of the precore/core gene of hepatitis $B$ virus genotypes $E$ and $A$ in West Africa: new subtypes, mixed infections and recombinations. J Gen Virol 87:1163-1173

Olokoba AB, Salawu FK, Danburam A (2011) Hepatitis B virus infection amongst pregnant women in North-Eastern Nigeria- A call for action. Niger J Clin Pract 14(1):10-13

Orito E, Mizokami M, Ina Y, Moriyama EN, Kameshima N, Yamamoto M, Gojobori $T$ (1989) Host-independent evolution and a genetic classification of the hepadnavirus family based on nucleotide sequences. Proc Natl Acad Sci U S A 86:7059-7062

Perz JF, Armstrong GL, Farrington LA, Hutin YJ, Bell BP (2006) The contribution of hepatitis $B$ virus and hepatitis $C$ virus infections to cirrhosis and primary liver cancer worldwide. J Hepatol 45:529-538

Poovorawan Y, Sanpavat S, Pongpunlert W, Chumdermpadetsuk S, Sentrakul P, Safary A (1989) Protective efficacy of a recombinant DNA hepatitis B vaccine in neonates of HBe antigen-positive mothers. JAMA 261:3278-3281

Poovorawan Y, Chongsrisawat V, Theamboonlers A, Leroux-Roels G, Kuriyakose S, Leyssen M, Jacquet J-M (2011) Evidence of protection against clinical and chronic hepatitis B infection 20 years after infant vaccination in a high endemicity region. J Viral Hepat 18:369-375

Prange R, Streeck RE (1995) Novel transmembrane topology of the hepatitis B virus envelope proteins. EMBO J 14(2):247-256

Ramezani F, Norouzi M, Sarizade GR, Poortahmasebi V, Kalantar E, Magnius L, Norder H, Domingo E, Jazayeri SM (2013) Mutation Hot Spots in Hepatitis B Surface Antigen in Chronic Carriers from Khoozestan Province, Southern of Iran. Iran J Allergy Asthma Immunol 12(3):269-275

Sadoh AE, Eregie CO (2008) Age at presentation for infant immunization in Nigeria: implications for hepatitis B immunization. Public Health 122:1318-1320

Schaefer S (2007) Hepatitis B, virus taxonomy and hepatitis B virus genotypes. World J Gastroenterol 13:14-21

Su WJ, Liu CC, Liu DP, Chen SF, Huang JJ, Chan TC, Chang MH (2012) Effect of age on the incidence of acute hepatitis $B$ after 25 years of a universal newborn hepatitis B immunization program in Taiwan. J Infect Dis 205:757-762

Tamura K, Peterson D, Peterson N, Stecher G, Nei M, Kumar S (2011) MEGA5: molecular evolutionary genetics analysis using maximum likelihood, evolutionary distance, and maximum parsimony methods. Mol Biol Evol 28:2731-2739

Theamboonlers A, Chongsrisawat V, Jantaradsamee P, Poovorawan Y (2001) Variants within the "a" determinant of HBsAg in children and adolescents with and without hepatitis B vaccination as part of Thailand's Expanded Program on Immunization (EPI). Tohoku J Exp Med 193:197-205

WHO (2005a) WHO vaccine preventable diseases monitoring system, 2005 global summary Immunization profile-Nigeria

WHO (2005b) Country stories. http://www.who.int/3by5/June2005_countryfacts. pdf?ua=1

World Health Organization (2009) Hepatitis B vaccines. Weekly epidemiological record., pp 20-405 